\title{
Entwicklungspolitischer Nutzen der schweizerische Entschuldungsfazilität : Zwischenbilanz oder abschliessendes Urteil?
}

Rolf Kappel

\section{(2) OpenEdition \\ Journals}

Édition électronique

URL : http://journals.openedition.org/sjep/1440

DOI : 10.4000/sjep.1440

ISSN : 1663-9677

Éditeur

Institut de hautes études internationales et du développement

Édition imprimée

Date de publication : 1 janvier 1994

Pagination : 298-301

ISSN : 1660-5926

Référence électronique

Rolf Kappel, « Entwicklungspolitischer Nutzen der schweizerische Entschuldungsfazilität

Zwischenbilanz oder abschliessendes Urteil ? ", Schweizerisches Jahrbuch für Entwicklungspolitik [En

ligne], 13 | 1994, mis en ligne le 10 juin 2013, consulté le 08 septembre 2020. URL : http://

journals.openedition.org/sjep/1440; DOI : https://doi.org/10.4000/sjep.1440 


\section{Entwicklungspolitischer Nutzen der schweizerische Entschuldungsfazilität: Zwischenbilanz oder abschliessendes Urteil?}

Rolf Kappel

Die hochverschuldeten Entwicklungsländer mit niedrigem Einkommen (SILICs) sind nach wie vor besondere Sorgenkinder der Entwicklungszusammenarbeit. Die Schweiz hat 1991 für diese Länder eine Entschuldungsfazilität eingerichtet, die für sich allein betrachtet und nur mit Blick auf die unmittelbaren Auswirkungen stark kritisiert werden kann. Einige Kritiker, wie beispielsweise Egli mit seinen Diskussionsbeiträgen, laufen jedoch Gefahr, durch eine enge Optik und nach den Erkenntnissen und Erfahrungen aus nicht einmal drei Jahren bereits ein endgültiges Urteil zu fällen.

Es ist unbestreitbar und unbestritten, dass die Rückkaufoperationen des BAWI im Vergleich zu den Gesamtschulden der betreffenden Entwicklungsländer nur geringes Gewicht haben, und den Schuldnerländern zunächst auch keine Zahlungserleichterungen bringen. Ebenso sind die erzielten Fortschritte bei der Einrichtung von Gegenwertfonds bislang ziemlich bescheiden, und solche Fonds mit Blick auf die gesamte Finanzpolitik der betreffenden Länder grundsätzlich nicht unproblematisch. Aus diesen ernüchternden Feststellungen kann man die Fazilität jedoch nicht abschliessend als Fehlschlag beurteilen.

Die Entschuldungsfazilität der Schweiz ist im Zusammenhang mit den seit 1988 deutlich zunehmenden multilateralen und bilateralen Massnahmen zur Schuldenerleichterung zu sehen. Dabei ist offenkundig, dass eine kritische Masse nur durch ein international koordiniertes Vorgehen zu erreichen ist, und es zeichnet sich ab, dass bilaterale und multilaterale Institutionen für die SILICs neue Anstrengungen zum Abbau der Schuldenbestände unternehmen werden. Sollte diese Entwicklung eintreten, wäre der Schweiz eine gewisse Vorreiterrolle unter den bilateralen Gebern nicht abzusprechen.

Welche Erwartungen knüpft man an solche Massnahmen? Im wesentlichen stehen vier Auswirkungen im Vordergrund:

1. Die Reduktion von Unsicherheiten für private in- und ausländische Investoren, in der Gegenwart und in der Zukunft.

2. Der kostengünstigere und erweiterte Zugang zu Handelskrediten.

3. Die Befreiung der betreffenden Regierungen und Verwaltungen von sehr aufwendigen Umschuldungsverhandlungen.

4. Die Wiederherstellung geregelter Beziehungen zu öffentlichen und privaten Gläubigern und damit der erneute Zugang zu Investitionskrediten.

Für jede dieser erwarteten Wirkungen gibt es bislang nur mehr oder minder umstrittene theoretische und empirische Begründungen und Belege. Ausserdem 
darf keinesfalls vernachlässigt werden, dass die nicht mehr vollständig bedienten Aussenschulden nur eines von vielen Problemen darstellen, mit denen sich die SILICs konfrontiert sehen: Makroökonomische Ungleichgewichte, grosse Defizite im Bildungs- und Ausbildungsstand des Arbeitskräftepotentials, eine schwache Infrastruktur, schlechte Wirtschaftspolitik, schwache Institutionen und Administrationen sowie politische Instabilitäten mögen als Schlagworte genügen. Schuldenreduktionen sind somit nur eine Massnahme zur Unterstützung umfangreicher, anspruchsvoller und langfristiger Reformprogramme.

Vor diesem Hintergrund ist die von Egli formulierte Kritik an der schweizerischen Entschuldungsfazilität zu diskutieren, und ein Schritt in die von ihm verwendete theoretische Rechenwelt ist dazu unumgänglich. Zunächst soll Eglis Rechenbeispiel eines Landes mit Gesamtschulden von 100 dahingehend modifiziert werden, dass das Land öffentlichen Gläubigern 80 und privaten Gläubigern 20 schuldet. Dies entspricht in stilisierter Vereinfachung den Verhältnissen der SILICs. Bei einem Zinssatz von 10\% und unendlicher Laufzeit der Schuld müssten jährlich Zahlungen von 10 erfolgen; das Schuldnerland zahlt jedoch nur 5, davon 4 an die öffentlichen und 1 an die privaten Gläubiger.

Nun sei angenommen, dass die öffentlichen Gläubiger einen Schuldenerlass beschliessen, der das Land wieder zur vollen Zahlungsfähigkeit zurückbringt. Die Kosten dieses Forderungsverzichts betragen offenkundig 50. Gleichzeitig hat als Nebeneffekt ein "Bail Out" der privaten Gläubiger stattgefunden, deren Forderungen nun wieder zu $100 \%$ bedient werden. Sie haben durch diese Operation 10 gewonnen.

Die öffentlichen Gläubiger können aber vor ihrem Schuldenerlass die privaten Forderungen vollständig aufkaufen. Dazu müssen sie den privaten Gläubigern mindestens 16 bezahlen, da - wie Egli zurecht betont - mit abnehmender Schuld der Wert der verbleibenden Forderungen ansteigt. Gegenüber der Situation vor dem Rückkauf würden die privaten Gläubiger somit 6 gewinnen, bzw. $37.5 \%$ der von den öffentlichen Gläubigern zum Rückkauf aufgewendeten Mittel würden als Gewinne in den Taschen der privaten Gläubiger verschwinden. Dies prangert Egli als nicht zu rechtfertigenden Nebeneffekt an - aber die Geschichte ist ja noch nicht zu Ende.

Nach dem Schuldenrückkauf kommt es zum Schuldenerlass der öffentlichen Gläubiger, der offenkundig weitere 30 kostet. Damit wird der Vorteil dieser Vorgehensweise gegenüber dem vorangehend geschilderten Erlass sichtbar. Im ersten Fall haben die öffentlichen Gläubiger Kosten von 50 und die privaten Gläubiger Gewinne von 10; bei der Variante mit Rückkauf betragen die Kosten 46 und die privaten Gläubiger gewinnen nur 6. Der Grund für den Unterschied ist offenkundig: Die privaten Gläubiger können durch den Rückkauf nicht in vollem Umfang vom öffentlichen Schuldenabbau als Trittbrettfahrer profitieren. Haben sich die öffentlichen Gläubiger einmal zum Schuldenerlass entschieden, ist es für sie kostengünstiger, den Rückkauf privater Forderungen vorzuziehen. Dieser Aspekt wird von Egli völlig vernachlässigt.

(Man kann auf diese Weise auch die Wirkung des von Egli als Alternative vorgeschlagenen Geschenks analysieren - das Ergebnis ist dasselbe. Im übrigen 
sei bemerkt, dass der Nettoressourcenzufluss an die SILICs seit Beginn der 90er Jahre zu mehr als 75 \% in Form von Kapitalschenkungen erfolgt, im Jahr 1993 in der Höhe von $15.1 \mathrm{Mrd}$ \$.)

Die Lehre aus dem Rechenbeispiel ist so simpel wie die Rechnerei selbst. Gleichgültig ob die Rückkehr zur vollständigen Zahlungsfähigkeit des Schuldnerlandes mit Schuldenerlassen oder Geschenken gefördert wird, die Kosten der öffentlichen Gläubiger und die Trittbrettfahrergewinne der privaten Gläubiger sind um so höher, je höher die Forderungen der privaten Gläubiger liegen. Der möglichst frühe Rückkauf privater Forderungen mit öffentlichen Mitteln ist somit effizient, da er diesen unvermeidbaren Nebeneffekt reduziert.

Egli postuliert, dass Schuldenkäufe Transaktionskosten verursachen, die bei Geschenken nicht anfallen. Dies ist natürlich eine verfehlte Annahme, wenn man - realistischerweise - berücksichtigt, dass mit den Geschenken Politikauflagen verbunden sind, die vertraglich vereinbart und kontrolliert werden müssen. Die Geschenke sollen ja zur Umschichtung und Steigerung der Produktion handelbarer Güter verwendet, und nicht für Konsumgüter verpulvert werden. Darüber hinaus sind die zuvor angesprochenen Transaktionskosten in Betracht zu ziehen, die mit den Umschuldungsverhandlungen anfallen. Die Weltbank verweist in der neuesten Ausgabe ihrer "World Debt Tables" zurecht darauf, dass diese Verhandlungen gerade in den SILICs ständig kompetente Fachleute absorbieren, die man für eine möglichst effiziente Implementation der Reformprogramme dringend benötigen würde.

Angesichts der unsicheren und teilweise widersprüchlichen Ergebnisse empirischer Untersuchungen bezweifelt Egli, dass ein Abbau der Schulden die Investitionstätigkeit positiv beeinflussen würde. In der Tat sollte man die mögliche Wirkung auf die Investitionen keinesfalls überschätzen, da nach dem gegenwärtigen Kenntnisstand die private Investititonstätigkeit in vielen Entwicklungsländern trotz aller Reformbemühungen aus mehreren Gründen bislang enttäuschend ist. Aber kann man denn ernsthaft in Zweifel ziehen, dass die mit dem nicht mehr bedienten Schuldenbestand verbundenen Unsicherheiten über die künftige Fiskalpolitik, die Wechselkursentwicklung und mögliche wirtschaftliche Instabilitäten die private Investitionstätigkeit hemmen? Die ökonomische Theorie und die Literatur zur ansteigenden Staatsverschuldung in einigen Industrieländern legen da ganz andere Erwartungen nahe.

Eine Zwischenbilanz über den entwicklungspolitischen Nutzen der schweizerischen Entschuldungsfazilität muss zum gegenwärtigen Zeitpunkt somit sehr bescheiden ausfallen. Ob es uns gefällt oder nicht: Die entwicklungsfördernde Wirkung dieses Instruments liegt noch weitgehend in der Zukunft. In welchem Umfang sie anfallen wird, hängt einerseits von künftigen Entscheidungen der internationalen Gebergemeinschaft ab (Wird eine kritische Masse beim Schuldenabbau der SILICs erreicht?), andererseits von den Massnahmen der Entwicklungszusammenarbeit und der Regierungen in den Schuldnerländern insgesamt (Greifen die mit Neugeld, Schuldenerleichterungen und technischer Zusammenarbeit gestützten Reformen? Gelingt die Umschichtung öffentlicher Mittel, unter anderem durch Gegenwertfonds, zur stärkeren Bekämpfung der Armut?). Nachdem die 
Schweiz mit der Einrichtung einer bilateralen Entschuldungsfazilität die Übernahme einer gewissen Vorreiterrolle gewagt hat, sollte sie die zunehmende Einsicht und Bereitschaft anderer Geber tatkräftig fördern, Instrumente zum konditionierten Schuldenabbau - einschliesslich Rückkauf - ebenfalls vermehrt zu nutzen. Aus begründbarer langfristiger Sicht spricht mehr dafür als dagegen.

\section{Literatur}

Vittorio Corbo, Stanley Fischer, Steven B. Webb (Eds., 1992): Adjustment Lending Revisited. Policies to Restore Growth; The World Bank, Washington D.C.

Dominik Egli, Marco Ferroni und Roger Denzer, Rolf Kappel, Oliver Landmann (1993) Die schweizerische Entschuldungspolitik: Flop oder Vorbild? Diskussionspapier Nr. 11, NFP 28, Bern

Paul Krugman (1988): Financing vs Forgiving a Debt Overhang; Journal of Development Economics, Vol. 29, No. 3

Jeffrey Sachs (1988): The Debt Overhang of Developing Countries, in: P. Findlay (Ed.): Debt, Growth and Stabilization. Essays in Memory of Carlos Diaz Alejandro; New York, Oxford

Weltbank (1993): World Debt Tables 1993-94, Vol. I and II; Washington D.C. 